\title{
Classical polarimetric method revisited to analyse the modulation capabilities of parallel aligned liquid crystal on silicon displays
}

\author{
A. Márquez ${ }^{1,2, *}$, F. J. Martínez ${ }^{1,2}$, S. Gallego ${ }^{1,2}$, M. Ortuño ${ }^{1,2}$, J. Francés ${ }^{1,2}$, A. Beléndez ${ }^{1,2}$, I. \\ Pascual $^{2,3}$ \\ ${ }^{1}$ Dept. de Física, Ing. de Sistemas y T. Señal, Univ. de Alicante, Ap. 99, E-03080, Alicante, Spain \\ ${ }^{2}$ I.U. Física Aplicada a las Ciencias y las Tecnologías U. de Alicante, Ap. 99, E-03080, Alicante, Spain \\ ${ }^{3}$ Dept. de Óptica, Farmacología y Anatomía, Univ. de Alicante, Ap. 99, E-03080, Alicante, Spain
}

\begin{abstract}
Novel liquid crystal on silicon displays (LCOS) technology has found widespread use in a number of applications dealing with the spatial modulation of the properties of a light wavefront. Parallel aligned LCoS (PA-LCoS) are especially interesting since they provide phase-only modulation with no coupling of amplitude modulation. However, typically LCoS are digitally addressed and this has been proven as a drawback since it causes fluctuations in the phase modulation. In this work we concentrate on PA-LCoS. We analyse the application of a classical polarimetric method for retardance measurement, which makes use of only linearly polarized light, typically used in wave plates. We analyse the effect of phase fluctuations on the measurements and provide a refinement of the method which allows estimating both the average retardance and the magnitude of the phase fluctuation. We demonstrate both theoretically and experimental that this extension of a classical method is both simple and very well suited for the measurement of the modulation properties of novel PA-LCoS.
\end{abstract}

Keywords: Liquid cristal on silicon displays, parallel aligned, retardance measurement, phase-only modulation, spatial light modulation, flicker, diffractive optics.

\section{INTRODUCTION}

Liquid crystal (LC) microdisplays have become a central device in a wide range of applications requiring spatial modulation of a wavefront like in diffractive optics ${ }^{[1]}$, optical storage ${ }^{[2]}$, or optical metrology ${ }^{[3]}$. Liquid crystal on silicon (LCoS) displays have become the most attractive microdisplays for these applications due to their very high spatial resolution and very high light efficiency ${ }^{[4][5]}$. However, several authors ${ }^{[6]-[10]}$ have detected that LCoS displays produce certain amount of phase flicker and/or depolarization. Among the different LCoS technologies typically available, parallel aligned LCoS (PA-LCoS) are especially interesting since they allow easy operation as phase-only devices without coupled amplitude modulation. In this case, no depolarization operation is readily available, however phase fluctuations are still a feature in digitally addressed LCoS devices ${ }^{[11]}$.

Different measurement methods have been proposed and demonstrated to characterize the phase-shift versus voltage in LCoS devices ${ }^{[12]-[14]}$. Some of these methods provide full polarimetric characterization and are based on Stokes-Mueller polarimeters ${ }^{[12][13]}$. Other methods are especifically oriented to obtain phase-shift modulation values such as the diffractive and the interferometric methods described in ref. ${ }^{[12][13]}$ : the first method is very well suited for instantaneous values measurement, whereas the latter is appropriate to obtain the average values. These methods can be applied to LCDs and LCoS devices in general. In ref. ${ }^{[14]}$ it was demonstrated that the addition of a high speed CCD camera and a Twyman-Green interferometer allows obtaining the instantaneous phase-shift modulation across the surface of an LCoS.

In the case of parallel aligned devices, they are totally characterized by their linear retardance vs. voltage values. Additional methods typically used in the characterization of waveplates become available to measure the linear retardance. The most popular are ellipsometric, polarimetric and interferometric ones. In interferometric methods we

Optics and Photonics for Information Processing VI, edited by Abdul A. S. Awwal, Khan M. Iftekharuddin, Proc. of SPIE Vol. 8498, 84980L · @ 2012 SPIE · CCC code: 0277-786/12/\$18 - doi: 10.1117/12.929677 
need a device introducing a carrier reference signal (heterodyne interferometry) such as a He-Ne Zeeman laser ${ }^{[15]}$ or an electrooptic light modulator ${ }^{[16]}$ working in the common-path geometry ${ }^{[17]}$. We can also have some element producing a phase-shift between the ordinary and extraordinary components of a beam (phase-shifting interferometry) such as a birefringent wedge ${ }^{[18]}$. Polarimetric methods of a high precision generally rely on null measurements, such as in the Senarmont compensator ${ }^{[19][20]}$. Other methods are based on the variation of the intensity when we change the angle of the elements in the polarimeter ${ }^{[2]-[25]}$. One common property to all these methods is that they assume that the birefringence in the wave plate has a constant value, no fluctuations, during the measurement process.

In the present work we want to propose a simple and easily available method to measure the retardance in a birefringent device exhibiting instabilities in the retardance. This is the actual case with PA-LCoS devices. First, in Section 2 we show the basic theory of the method proposed, which is an extended version of probably the technique most utilized in retardance measurement in waveplates. We show the degree of the deviations that can be expected when there are fluctuations in the retardance. We also analyze how this method can be adapted to measure both the average retardance value and its fluctuation amplitude. In Section 3 we proceed with the measurements and with the analysis of the results. Validation of the proposed method is accomplished through comparison with instantaneous retardance measurements obtained applying the classical polarimetric technique but appropriately adapted for real-time operation. Eventually, the main conclusions are given in Section 4.

\section{THEORETICAL DEVELOPMENT}

First, let us define the retardance $\Gamma$ of the wave plate:

$$
\Gamma=\frac{2 \pi \Delta n(\lambda) d}{\lambda}
$$

$\lambda$ is the wavelength of the incident light, $\Delta \mathrm{n}(\lambda)$ is the difference between the extraordinary and the ordinary index of the birefringent material (e.g. the liquid crystal) at this wavelength, and $d$ is the thickness of the wave plate. We should distinguish this value, which is the total retardance of the waveplate, from the retardance value modulus $2 \pi$ extracted from most of the measurement methods. The relation between the total retardance $\Gamma$ and the retardance $\Gamma_{0}$ modulo $2 \pi$ is given by,

$$
\Gamma=\Gamma_{0}+m 2 \pi
$$

where $\mathrm{m}$ is an integer, called the order of the waveplate. In the case of the zero order wave plate $\mathrm{m}=0$, and $\Gamma_{0}=\Gamma$ which is actually the case in LCoS devices.

To measure the retardance of the wave plate we use the linear polarimeter ${ }^{[21]-[25]}$ (see Fig. 1) illuminated with a monochromatic laser source. In the basic configuration there is a radiometer at the output of the polarimeter.
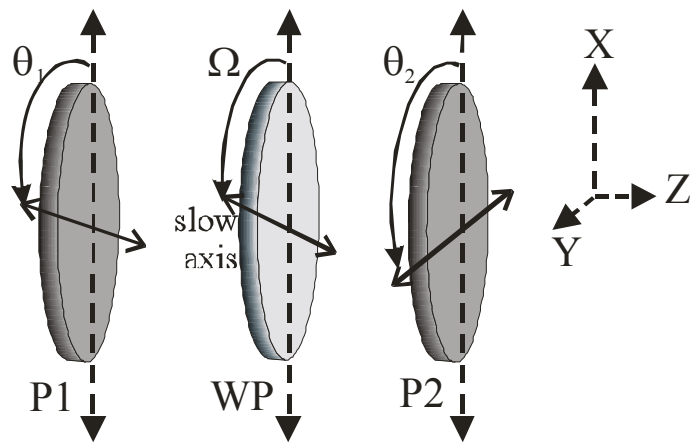

Figure 1. Linear polarimeter with the wave plate WP to be measured. P1 and P2 are the polarizers. 
Next we follow the procedure in ref. ${ }^{[25]}$ to obtain the expressions for the intensity transmission through a linear polarimeter as a function of the orientation of the polarizers and the orientation and retardance of our sample, i.e. the waveplate. We use the Jones matrix formalism. In this formalism the matrix $\mathrm{W}(\Gamma)$ for a wave plate of retardance $\Gamma$, and the matrix $\mathrm{P}_{\mathrm{X}}$ for a linear polarizer with the transmission axis along the $\mathrm{X}$ axis, are given by,

$$
\begin{gathered}
W(\Gamma)=\left(\begin{array}{cc}
\exp (-i \Gamma / 2) & 0 \\
0 & \exp (i \Gamma / 2)
\end{array}\right) \\
P_{X}=\left(\begin{array}{ll}
1 & 0 \\
0 & 0
\end{array}\right)
\end{gathered}
$$

The rotation matrix $\mathrm{R}(\mathrm{w})$ for an angle $\mathrm{w}$ is,

$$
R(w)=\left(\begin{array}{cc}
\cos w & \sin w \\
-\sin w & \cos w
\end{array}\right)
$$

According to this notation, the complex amplitude of the electric field $\vec{E}_{\text {OUT }}$ at the output of the linear polarimeter in Figure 1 results from the product,

$$
\vec{E}_{\mathrm{OUT}}=P_{X} R\left(+\theta_{2}\right) R(-\Omega) W_{0}(\Gamma) R(+\Omega)\left(\begin{array}{c}
\cos \left(\theta_{1}\right) \\
\sin \left(\theta_{1}\right)
\end{array}\right)
$$

and the intensity transmission $\mathrm{I}_{\mathrm{OUT}}$ is calculated as the Hermitian scalar product,

$$
I_{\text {OUT }}=\vec{E}_{\text {OUT }}^{\dagger} \cdot \vec{E}_{\text {OUT }}
$$

where $\vec{E}_{\text {OUT }}^{\dagger}$ is the Hermitian conjugate of the vector $\vec{E}_{\text {OUT }}$.

$$
I_{\text {OUT }}=\frac{I_{0}}{2}\left[1+\cos \left[2\left(\theta_{2}-\Omega\right)\right] \cos \left[2\left(\theta_{1}-\Omega\right)\right]+\cos \Gamma \sin \left[2\left(\theta_{2}-\Omega\right)\right] \sin \left[2\left(\theta_{1}-\Omega\right)\right]\right]
$$

where we take into account the incident intensity $\mathrm{I}_{0}$. Let us rewrite this expression in a more convenient form,

$$
I_{\text {OUT }}=\frac{I_{0}}{2}[1+A+B \cos \Gamma]
$$

where

$$
\begin{aligned}
& A=\cos \left[2\left(\theta_{2}-\Omega\right)\right] \cos \left[2\left(\theta_{1}-\Omega\right)\right] \\
& B=\sin \left[2\left(\theta_{2}-\Omega\right)\right] \sin \left[2\left(\theta_{1}-\Omega\right)\right]
\end{aligned}
$$

In general, for a fixed position of polarizers and sample wave plate, a fluctuation in the retardance $\Delta \Gamma$ would produce an intensity variation $\Delta \mathrm{I}$. The two fluctuations are related through the first derivative of the intensity with respect to the retardance, i.e.,

$$
\Delta I_{\text {OUT }}=\left|\frac{d I_{\text {OUT }}}{d \Gamma}\right| \Delta \Gamma
$$


where we consider the absolute value of the derivative. If we invert the relation we can calculate the error $\Delta \Gamma$ due to a deviation $\Delta \mathrm{I}$ in the measurement of the intensity, which can be due to any source of error or tolerances in the system such as deviations in the angular orientation of the polarizers in the setup just to mention a possibility. Thus, we have

$$
\Delta \Gamma=\left|\frac{d I_{\text {OUT }}}{d \Gamma}\right|^{-1} \Delta I_{\text {OUT }}
$$

And if we calculate this expression we obtain,

$$
\Delta \Gamma=\left|\frac{2}{B \sin \Gamma}\right| \frac{\Delta I_{\text {OUT }}}{I_{O}}
$$

Let us analyse eq. (13). The deviation is minimum when the sine is close to unity, i.e. when $\Gamma$ approaches $\pm \pi / 2$, and diverges when the sine approaches zero, i.e. when $\Gamma$ is close to 0 or $\pi$. Thus, deviations in $\Delta \Gamma$ depend strongly on the existing value of the retardance $\Gamma$. Furthermore, to reduce $\Delta \Gamma$ we should increase the value of the parameter B. Therefore, if we look at eq. (10) we should have the following orientations of the polarization elements,

$$
\left(\theta_{2}-\Omega\right)= \pm\left(\theta_{1}-\Omega\right)= \pm \pi / 4
$$

This means that the polarizers should be oriented at $\pm 45^{\circ}$ with respect to the slow axis of the wave plate. The polarizers can still be parallel or perpendicular each other, thus producing respectively the following intensity measurements,

$$
\begin{aligned}
& I_{\text {OUT }}^{\prime \prime}=\frac{I_{0}}{2}[1+\cos \Gamma] \\
& I_{\text {OUT }}^{\perp}=\frac{I_{0}}{2}[1-\cos \Gamma]
\end{aligned}
$$

These two measurements are probably the most typically used to obtain the retardance in linear retarder devices. Next, we want to analyze their validity in case there are instabilities or fluctuations in the linear retardance, as it is the case in digitally addressed LCoS devices ${ }^{[12]-[14]}$. As a first approximation we may consider a triangular profile for the periodic variation of retardance with time $\Gamma(t)$, as shown in Fig. 2, with an average value $\bar{\Gamma}$, retardance fluctuation amplitude $a$, and period $T$, i.e. described by the following equation,

$$
\Gamma(t)= \begin{cases}\bar{\Gamma}-a+\frac{2 a}{T / 2} t & 0 \leq t<T / 2 \\ \bar{\Gamma}+3 a-\frac{2 a}{T / 2} t & T / 2 \leq t<T\end{cases}
$$

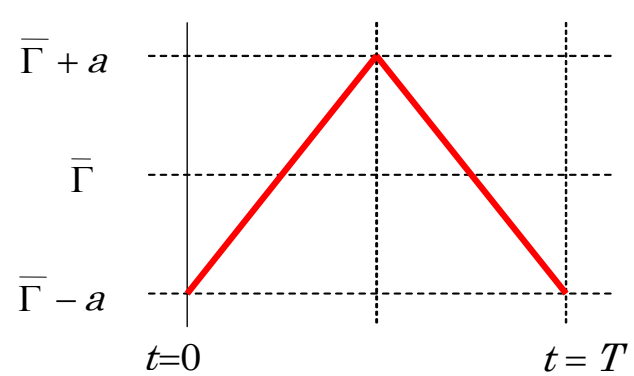

Figure 2. Triangular profile considered for the temporal fluctuation of the linear retardance. 
To calculate the average value in Eq. (15) and (16) we simply need to consider one semiperiod. The average for the cosine term is given by the next sequence of operations,

$$
\left\langle\cos \left(\bar{\Gamma}-a+\frac{2 a}{T / 2} t\right)\right\rangle=\frac{1}{T / 2} \int_{0}^{T / 2} \cos \left(\bar{\Gamma}-a+\frac{2 a}{T / 2} t\right) d t=\left.\frac{1}{T / 2} \frac{\sin \left(\bar{\Gamma}-a+\frac{2 a}{T / 2} t\right)}{\frac{2 a}{T / 2}}\right|_{0} ^{T / 2}=\frac{\sin (\bar{\Gamma}+a)-\sin (\bar{\Gamma}-a)}{2 a}=\frac{\cos (\bar{\Gamma}) \sin (a)}{a}
$$

Then, Eqs. (15) and (16) may be rewritten in terms of the average value and the fluctuation amplitude as follows,

$$
\begin{aligned}
& \left\langle I_{\text {OUT }}^{\prime \prime}\right\rangle=\frac{I_{0}}{2}\left[1+\frac{\sin a}{a} \cos \bar{\Gamma}\right] \\
& \left\langle I_{\text {OUT }}^{\perp}\right\rangle=\frac{I_{0}}{2}\left[1-\frac{\sin a}{a} \cos \bar{\Gamma}\right]
\end{aligned}
$$

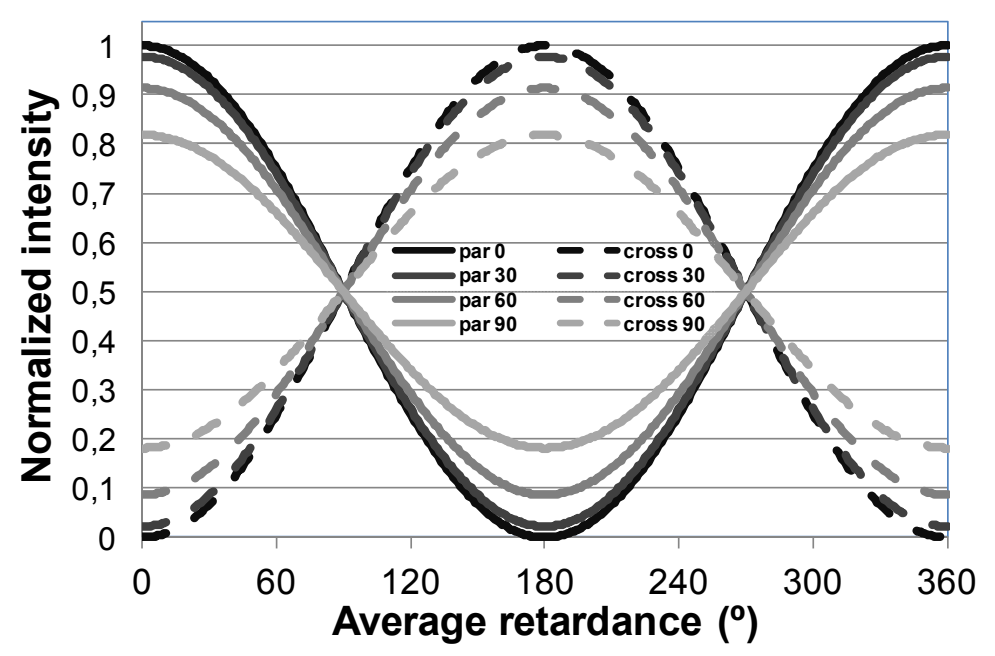

Figure 3. Simulation of the normalized intensity versus the average retardance measured for parallel (continuous) and crossed (dashed) polarizers and for various values of the fluctuation magnitude $a$, shown in the legend.

From these expressions it results a deviation from the classical expressions previously given in Eqs. (15) and (16), which become modulated by the presence of a sinc function dependent on the magnitude of the fluctuation $a$. In Figure 3 we represent the normalized intensity values given by Eqs. (19) and (20) as a function of the average retardance. Dashed and continuous curves correspond respectively to the simulation for crossed and parallel polarizers. The curves have been calculated for various magnitudes of the fluctuation $a$ ranging from $0^{\circ}$ (ideal) to $90^{\circ}$. When no retardance fluctuation is present the intensity reaches maxima and minima values respectively of 1 and 0 . As the retardance fluctuation increases the maxima and minima separates from these ideal values. The maxima and minima in the curves are produced at average values $\bar{\Gamma}$ which are multiple of $180^{\circ}$. At these points, from Eqs. (19) and (20), we obtain that the absolute difference in intensity, $I_{d i f f}$, with respect to the ideal $\left(a=0^{\circ}\right)$ is equal to,

$$
I_{\text {diff }}=0.5\left(1-\frac{\sin a}{a}\right)
$$

In Figure 4 (a) we represent $I_{\text {diff }}$ versus the magnitude of the fluctuation $a$, calculated using Eq. (21). We see the nonlinear increase of $I_{d i f f}$ with the increase of the fluctuation due to the $\operatorname{sinc}$ function. If this plot is reversed, Figure 4(b), 
we have a straightforward way to estimate the magnitude of the fluctuation simply by looking at the deviation in the intensity at the maxima or minima in the intensity, i.e. by selecting the points where the average value $\bar{\Gamma}$ is multiple of $180^{\circ}$. On the figure we show (dashed line) the polynomial fit to the curve, using a $6^{\text {th }}$ degree polynomial function. For the sake of completeness we show the squared correlation factor, which is very close to one. Using this polynomial we can quantitatively obtain the magnitude of the fluctuation from the value of $I_{\text {diff }}$.
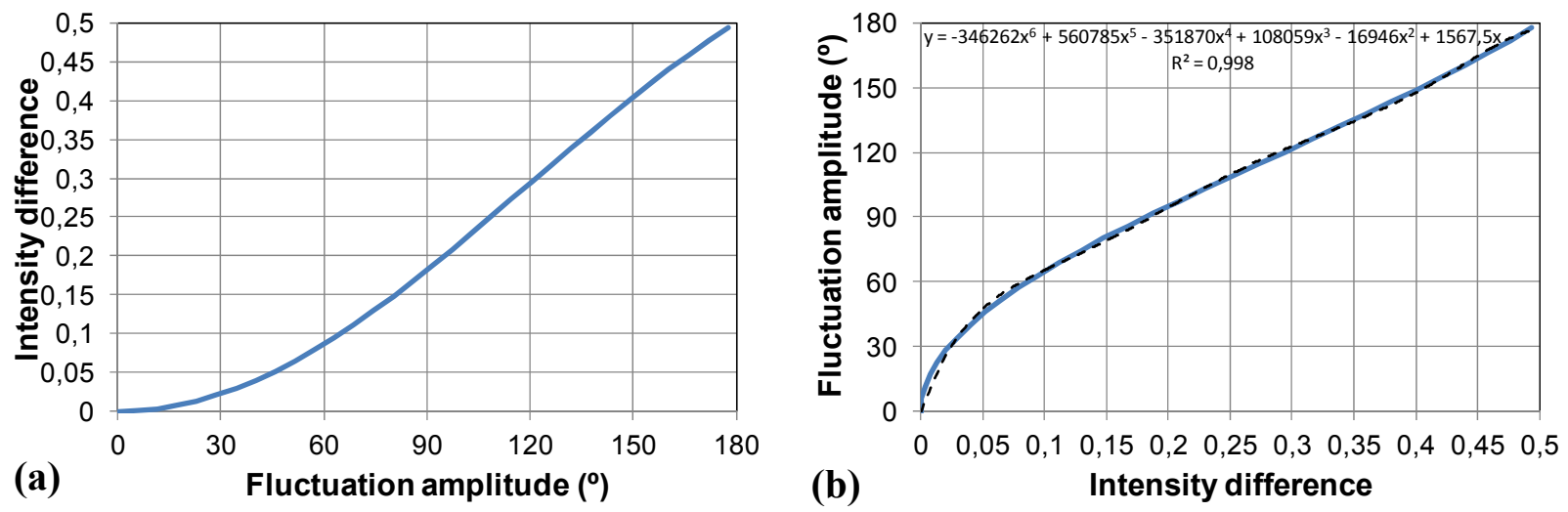

Figure 4. (a) Intensity difference (absolute value) with respect to the ideal $a=0^{\circ}$ as a function of the fluctuation amplitude, at the maxima and minima intensity points, occurring when the average value $\bar{\Gamma}$ is multiple of $180^{\circ}$; (b) Reverse relation, where a polynomial fit has been calculated with respect to the theoretical curve.

Let us now combine Eqs. (15) and (16) to remove the normalization factor to obtain the following expression,

$$
\frac{\left\langle I_{\text {OUT }}^{\prime \prime}\right\rangle-\left\langle I_{\text {OUT }}^{\perp}\right\rangle}{\left\langle I_{\text {OUT }}^{\prime \prime}\right\rangle+\left\langle I_{\text {OUT }}^{\perp}\right\rangle}=\frac{\sin a}{a} \cos \bar{\Gamma}
$$

In the case when no fluctuations exist $\left(a=0^{\circ}\right)$ we recover the classical result,

$$
\frac{\left\langle I_{\text {OUT }}^{\prime \prime}\right\rangle-\left\langle I_{\text {OUT }}^{\perp}\right\rangle}{\left\langle I_{\text {OUT }}^{\prime \prime}\right\rangle+\left\langle I_{\text {OUT }}^{\perp}\right\rangle}=\cos \bar{\Gamma}
$$

Since from Fig. 4(b) we have an estimation of the amplitude of the fluctuation $a$ then Eq. (22) can be inverted to obtain the average retardance $\bar{\Gamma}$,

$$
\bar{\Gamma}=\cos ^{-1}\left(\frac{\left(\left\langle I_{\text {OUT }}^{\prime \prime}\right\rangle-\left\langle I_{\text {OUT }}^{\perp}\right\rangle /\left\langle I_{\text {oUT }}^{\prime \prime}\right\rangle+\left\langle I_{\text {OUT }}^{\perp}\right\rangle\right)}{(\sin a / a)}\right)
$$

\section{RESULTS AND DISCUSSION}

In Figure 5 we show the experimental used in this work to measure obtain the average retardance versus applied voltage (gray level) for a parallel aligned LCoS device. It basically consists of a light source (He-Ne laser, $\lambda=633 \mathrm{~nm}$ ), the LCoS, the necessary input and output linear polarizers which are used in parallel or crossed configuration, and some intensity measuring device (radiometer). We also introduce two high quality non-polarizing cube beam splitters (model 10BC16NP.4, from Newport): one of them to separate the incident and reflected beams, and the other to enable amplitude division of the reflected beam so that crossed and parallel intensity can be measured simultaneously (thus, two 
radiometers are introduced). In some of the experiments the two radiometers are connected to the two channels of an oscilloscope enabling to obtain synchronized the instantaneous parallel and crossed polarizers intensity values. Therefore we are able to calculate the instantaneous value of the linear retardance as it will be shown. We note that a quarter wavelength waveplate is added after the laser so that enough light passes through the first polarizer irrespectively of the orientation of its transmission axis.

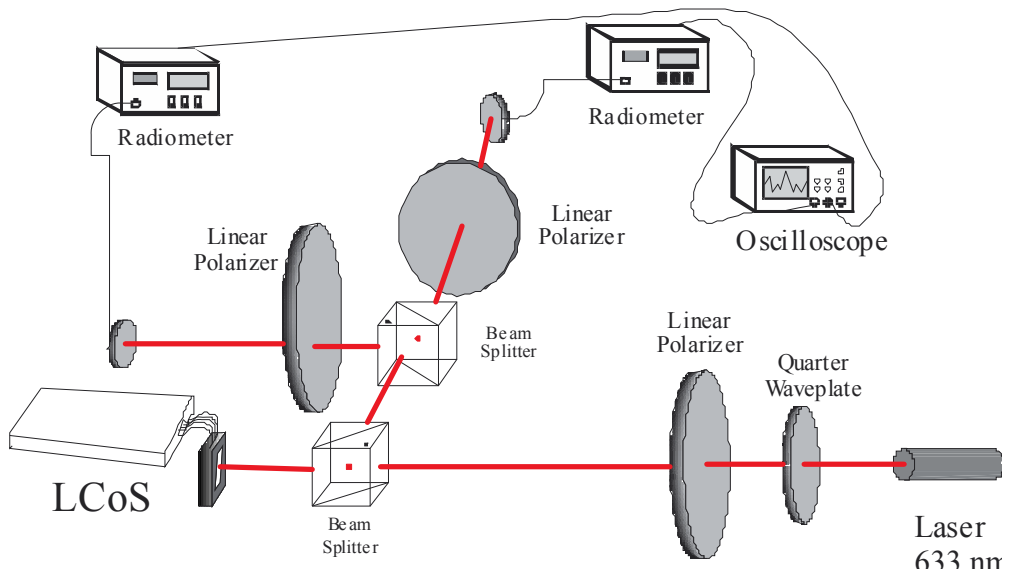

Figure 5. Experimental setup used to measure the linear retardance as a function of the applied voltage (gray level) for a PALCoS. The setup allows both to measure average and instantaneous values. Detailed description of the setup is given in the present section.

In this paper we analyze a phase-only LCoS. It corresponds to an electrically controlled birefringence (ECB) LCoS display distributed by the company HOLOEYE. It is an active matrix reflective mode device with 1920x1080 pixels and 0.7 " diagonal named the PLUTO Spatial Light Modulator (SLM). The pixel pitch is of $8.0 \mu \mathrm{m}$ and the display has a fill factor of $87 \%$. The signal is addressed via a standard DVI (Digital Visual Interface) signal. By means of the RS-232 interface and its corresponding provided software, we can perform gamma control to configure the modulator for different applications and wavelengths. Besides, different pulse width modulation (PWM) addressing schemes (digital addressing sequences) ${ }^{[26][27]}$ can be generated by the driver electronics, which can be programmed using the software included with the device. This will be applied in the present paper to characterize the magnitude of the fluctuation and the average retardance for various digital addressing sequences.

In particular we will evaluate two digital addressing sequences whose configuration files are provided with the software and which can be loaded using this device driver software. They correspond to the configurations labelled as "18-6 2pi linear $633 \mathrm{~nm}$ " and "5-5 2pi linear $633 \mathrm{~nm}$ ". The first number indicates the quantity of "equally weighted" bit-planes, and the second number the quantity of "binary" bit-planes ${ }^{[26]}$. This means that the sequence 18-6 is longer than the one corresponding for the sequence 5-5. In principle the shorter the sequence the smaller the flicker ${ }^{[26]}$. However, a larger sequence provides a larger number of possible phase levels: $(18+1) \times 2^{6}=1216$ for the sequence " $18-6$ " and $(5+1) \times$ $2^{5}=192$ for the sequence " $5-5$ ". In principle, the sequences " $18-6$ 2pi linear $633 \mathrm{~nm}$ " and " $5-52$ pi linear $633 \mathrm{~nm}$ " have been respectively optimized for the wavelength $633 \mathrm{~nm}$. The voltage depth and gamma for the sequence 18-6 and 5-5 are designed to provide a phase depth of $2 \pi$ radians, and quite a linear relation between phase value and gray level. Using these three different sequences we can evaluate different levels of flicker.

The input polarizer transmission axis is at $+45^{\circ}$ to the vertical of the lab, which is the $\mathrm{X}$-axis of our reference system as shown in Figure 1. The director for the liquid crystal (i.e. the extraordinary axis) in the LCoS is oriented along the horizontal, i.e. at $+90^{\circ}$ with respect to the X-axis. The director axis in nematic filled devices, as it is the case for our LCoS typically corresponds to the slow axis. Light impinges perpendicularly onto the LCoS and onto the two beamsplitters. Perpendicular incidence onto the beam-splitters is important so that they do not introduce polarization effects on their own. Typically the angular uncertainty on the orientation of the input and output polarizers with respect to the neutral lines of the LCoS can be considered $\pm 1^{\circ}$.

In Figure 6 we show the intensity measured for parallel (continuous) and crossed (dashed) polarizers as a function of gray level applied to the LCoS and for the two electrical sequences previously presented in this Section. This curve 
should be looked at keeping in mind Figure 3 corresponding to the simulations previously discussed. We see that both electrical sequences oscillate with the gray level with a slightly smaller amplitude in the case of the sequence "18-6 2pi linear $633 \mathrm{~nm}$ ", thus it possess a larger retardance fluctuation. The two sequences oscillate out of phase, with the sequence "18-6 2pi linear 633nm" reaching the extremals at lower gray levels. However at gray level 255 both sequences converge, thus they may show the same average retardance value in the end. We note the quantization steps in the case of sequence "5-5 2pi linear $633 \mathrm{~nm}$ " due to the limited number of levels which is smaller than 256 .

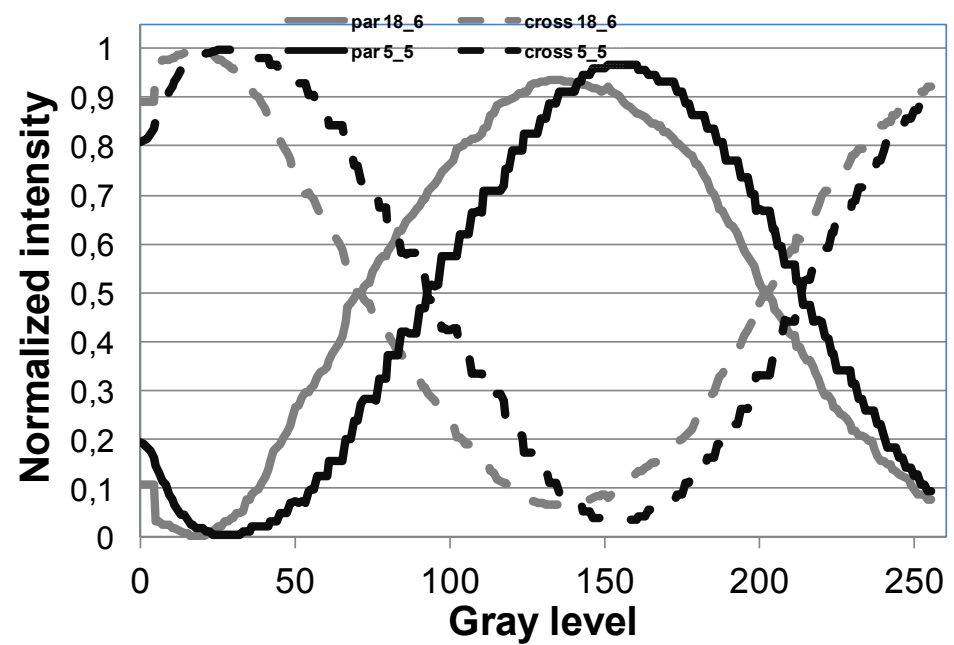

Figure 6. Experimental measurements of the normalized intensity versus the gray level measured for parallel (continuous) and crossed (dashed) polarizers and for the electrical sequences "18-6 2pi linear 633nm" and "5-5 2pi linear 633nm".

From the intensity values at the extremals and taking into account Figure 4(b) we obtain an estimation of the fluctuation amplitude, which we show in the third column in Table 1. We see that the first extremals in both sequences do not contain any distinguishable fluctuation. In the second extremal, sequence "18-6 2pi linear 633nm" contain a fluctuation amplitude about $54^{\circ}$ whereas sequence " $5-52$ pi linear $633 \mathrm{~nm}$ " stays at about $34^{\circ}$. Next we want to validate that these fluctuation amplitudes can be considered a good estimation of the actual fluctuation. To this goal we use the same setup in Fig. 5 but now the two radiometers are connected to the oscilloscope. This allows us to calculate the instantaneous retardance value using the classical expression in Eq. (23). In Figure 7 we show the temporal evolution of the retardance at gray levels close to the extremals and for the two sequences considered. We clearly appreciate the periodic evolution of the retardance with a characteristic pseudo triangular profile. The oscillation amplitude is larger for the sequence "186 2pi linear $633 \mathrm{~nm}$ ". We note that the results shown in Fig. 7 are wrapped values produced by the dominium of definition of the $\cos ^{-1}$ function. Actually half of the peaks should be folded against the 0 -ordinate axis producing an average retardance value close to zero. Taking this into account the fluctuation amplitude actually corresponds to the peak value in Fig. 7. This is the value considered in the fourth column in Table 1. Going back to Table 1, then we see that there is a good agreement between the fluctuation amplitude obtained with the method we propose and the one obtained from the instantaneous retardance values that we measure.

Table 1. Comparison between the fluctuation amplitude $a$ obtained with the method proposed in the paper ( $3^{\text {rd }}$ column), and the experimental value obtained from the instantaneous retardance values measured when connecting the two radiometers in the setup to the oscilloscope (4rth column).

\begin{tabular}{llll}
\hline Sequence & $I_{\text {diff }}$ & $\begin{array}{l}\text { Fluctuation Amplitude } \\
\text { (using Fig. 4(b)) }\end{array}$ & $\begin{array}{l}\text { Fluctuation Amplitude } \\
\text { (instantaneous values) }\end{array}$ \\
\hline $18 \_6$ & 0,07 & $54^{\circ}(\mathrm{GL} \mathrm{134)}$ & $46^{\circ}$ (GL 130) \\
$5 \_5$ & 0,03 & $34^{\circ}(\mathrm{GL} \mathrm{156)}$ & $32^{\circ}$ (GL 149) \\
\hline
\end{tabular}




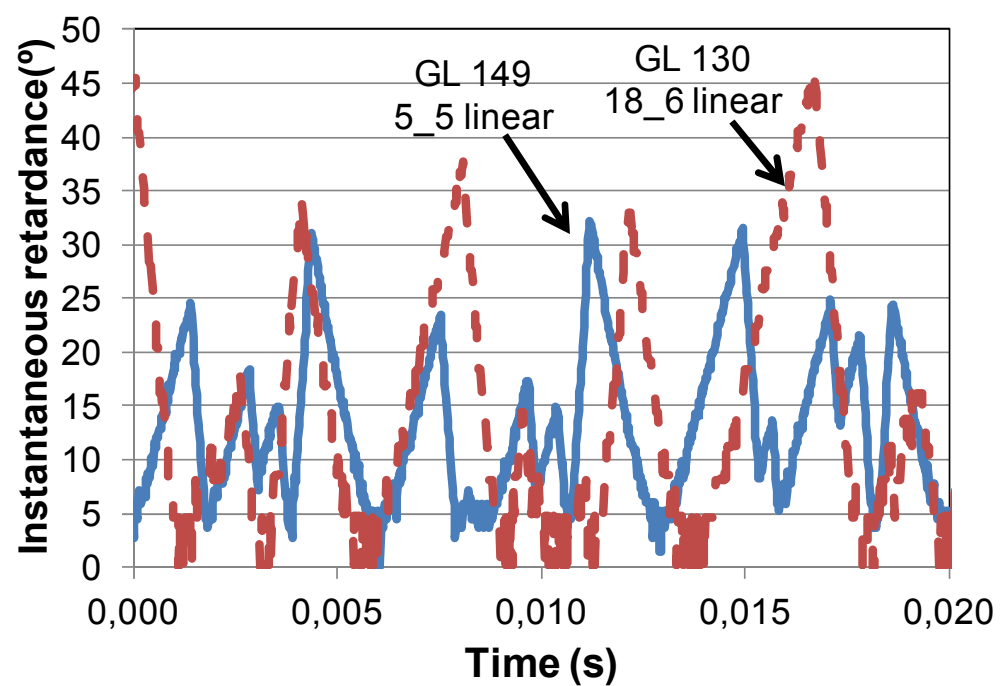

Figure 7. Temporal evolution of the retardance at gray levels, indicated on the figure, close to the extremals and for the two sequences considered "18-6 2pi linear 633nm" and "5-5 2pi linear 633nm".

Once the fluctuation amplitude values have been validated we can introduce them in Eq. (24) to calculate the average retardance. What we do is to extrapolate the fluctuation amplitude value calculated from the extremals to a wider gray level range: the fluctuation amplitude value from the first extremal is considered to be roughly valid until half of the distance between the two extremals, and from there the value from the second extremal is the one considered valid. We show in Fig. 8 (a) and (b), respectively for the sequences "18-6 2pi linear 633nm" and "5-5 2pi linear 633nm", the average retardance value calculated with this procedure as a function of the gray level. They correspond to "corrected" curve (continous line) label in the legend. The "uncorrected" is obtained when no fluctuations are considered, i.e. $a=0^{\circ}$. We see that deviations between the corrected and the uncorrected curves are only encountered in the intervals next to the extremals.

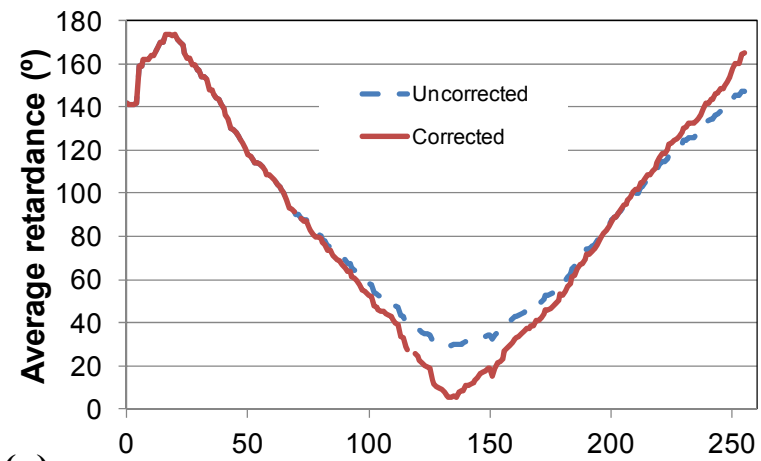

(a)

Gray level

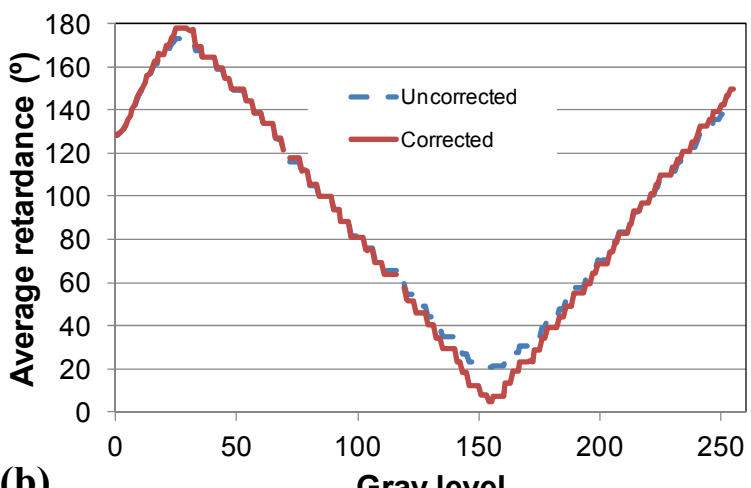

Figure 8. Average retardance (wrapped values) vs. gray level considering the existence of the fluctuation amplitude in the retardance, "corrected" curve, and considering the ideal $a=0^{\circ}$, "uncorrected" curve. (a) "18-6 2pi linear 633nm"; (b)"5-5 2pi linear 633nm".

In order to validate the average retardance values just shown we have proceeded with the measurement of the instantaneous retardance for a series of gray levels $(0,100,200$, and 255) using the procedure with the oscilloscope previously described in Fig. 7. We show the results in Fig. 9 (a) and (b) respectively for the sequences "18-6 2pi linear $633 \mathrm{~nm}$ " and "5-5 2pi linear 633nm". From these measurements we have elaborated Table 2, where the average retardance values are given. We also show the fluctuation amplitude value calculated as half the peak-to-peak amplitude of the temporal oscillation of the instantaneous retardance.

We see that the values for the average retardance show a very good agreement with the values in the Fig. 8 (a) and (b). The only significant deviations appear at gray level 255 , probably due to the fact that the curves are close to a third hypothetical extremal, thus the value used for the amplitude of fluctuation is losing its validity in this gray level range. 

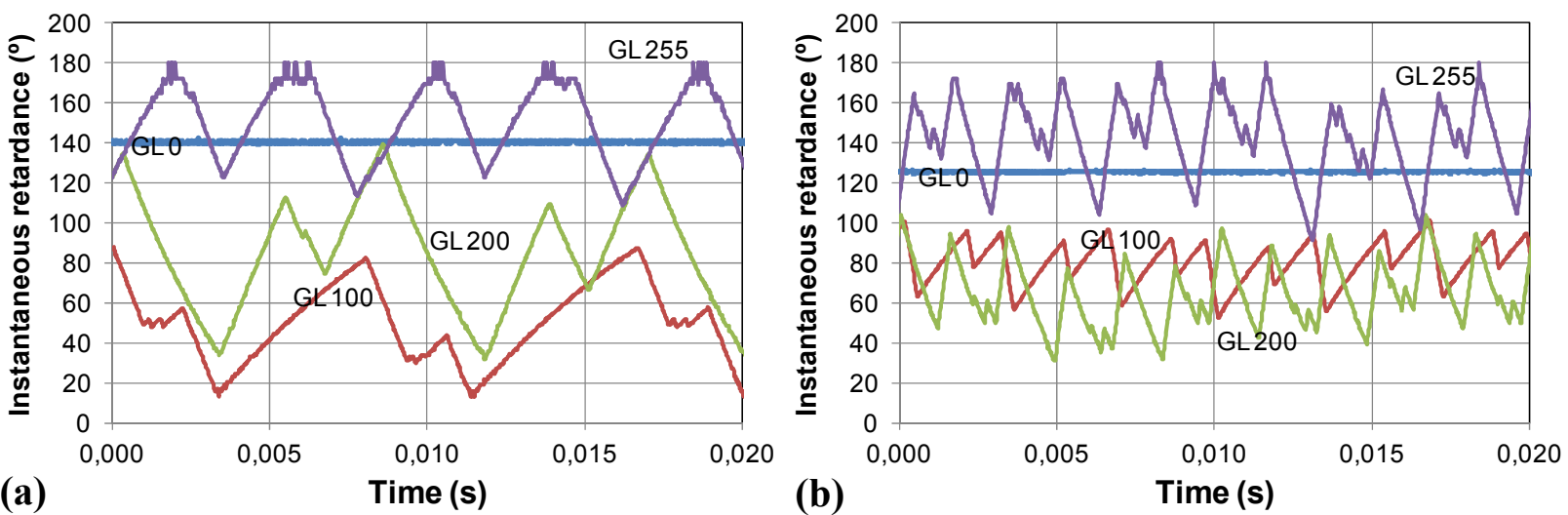

Figure 9. Temporal evolution of the retardance at gray levels $0,100,200$ and 255, indicated on the figure. (a) "18-6 2pi linear 633nm"; (b) "5-5 2pi linear 633nm".

Table 2. Values for the average retardance and the magnitude of the amplitude of its fluctuation extracted from the instantaneous retardance measurements shown in Figure 9.

\begin{tabular}{lccc}
\hline Sequence & Gray level & Avg. retardance & Fluctuation Amplitude \\
\hline $18 \_6$ & 0 & $141^{\circ}$ & $2^{\circ}$ \\
& 100 & $51^{\circ}$ & $37^{\circ}$ \\
& 200 & $86^{\circ}$ & $54^{\circ}$ \\
& 255 & $144^{\circ}$ & $36^{\circ}$ \\
$5 \_5$ & & $1^{\circ}$ \\
& 0 & $125^{\circ}$ & $24^{\circ}$ \\
& 100 & $77^{\circ}$ & $37^{\circ}$ \\
& 200 & $67^{\circ}$ & $44^{\circ}$ \\
\hline
\end{tabular}

\section{CONCLUSIONS}

We have demonstrated both theoretically and experimentally the feasibility of a novel technique which is suitable to obtain the retardance of linear retarders, such as parallel aligned LCoS displays, in the presence of instabilities or fluctuations in the retardance. The method is an extension a well known intensity-based polarimetric method. The method is both simple and does not require especial or expensive equipment. In a first step we calculate the value for the amplitude of the fluctuation in the retardance at specific points where the average retardance value is multiple of $180^{\circ}$. We note that validation of the technique has been accomplished by applying the classical intensity-based polarimetric method but with the appropriate modifications enabling instantaneous retardance measurements.

As a final remark we note that the two sequences considered in this work enable the LCoS to attain a dynamic retardance range of $360^{\circ}$. In one of the two cases, the electrical sequence " $5-52 \mathrm{pi}$ linear $633 \mathrm{~nm}$ " the amplitude of the fluctuations is basically below $30^{\circ}$. We note from the work of Lizana et al. ${ }^{[13]}$ (see for example Fig. 3(b) therein) that diffraction efficiency of a blazed grating with such fluctuations does not show a significant degradation since diffraction efficiency is still over $95 \%$. Therefore this electrical sequence may be a good choice for great many applications.

\section{ACKNOWLEDGEMENTS}

This work was supported by the Ministerio de Trabajo y Competitividad of Spain under projects FIS2011-29803-C02-01 and FIS2011-29803-C02-02 and by the Generalitat Valenciana of Spain (projects PROMETEO/2011/021 and ISIC/2012/013). 


\section{REFERENCES}

[1] J. Turunen and F. Wyrowski Edts., [Diffractive Optics for Industrial and Commercial Applications], Akademie Verlag, Berlin, (1997).

[2] H. J. Coufal, D. Psaltis and B. T. Sincerbox, Eds., [Holographic Data Storage], Springer-Verlag, Berlin, (2000).

[3] W. Osten, C. Kohler and J. Liesener, "Evaluation and application of spatial light modulators for optical metrology," Opt. Pura Apl. 38, 71-81 (2005).

[4] S. T. Wu and D. K. Yang, [Reflective Liquid Crystal Displays], John Wiley \& Sons Inc., Chichester, (2005).

[5] N. Collings, T. Davey, J. Christmas, D. Chu, and B. Crossland, "The Applications and Technology of Phase-Only Liquid Crystal on Silicon Devices”, J. Display Technol. 7, 112-119 (2011).

[6] J. E. Wolfe and R. A. Chipman, "Polarimetric characterization of liquid-crystal-on-silicon panels," Appl. Opt. 45, 1688-1703 (2006).

[7] A. Márquez, I. Moreno, C. Iemmi, A. Lizana, J. Campos and M. J. Yzuel, "Mueller-Stokes characterization and optimization of a liquid crystal on silicon display showing depolarization," Opt. Express 16, 1669-1685 (2008).

[8] P. Clemente, V. Durán, Ll. Martínez-León, V. Climent, E. Tajahuerce, and J. Lancis, "Use of polar decomposition of Mueller matrices for optimizing the phase response of a liquid-crystal-on-silicon display," Opt. Express 16, 1965-1974 (2008).

[9] A. Lizana, A. Márquez, I. Moreno, C. Iemmi, J. Campos and M. J. Yzuel, "Wavelength dependence of polarimetric and phase-shift characterization of a liquid crystal on silicon display," J. Eur. Opt. Soc. - Rapid Pub. 3, 08011 1-6 (2008).

[10]A. Lizana, I. Moreno, C. Iemmi, A. Márquez, J. Campos and M. J. Yzuel, "Time-resolved Mueller matrix analysis of a liquid crystal on silicon display," Appl. Opt. 47, 4267-4274 (2008).

[11] A. Lizana, I. Moreno, A. Márquez, E. Also, C. Iemmi, J. Campos and M.J.Yzuel, "Influence of the temporal fluctuations phenomena on the ECB LCoS performance," Proc. SPIE 7442, 74420G-1 (2009).

[12] A. Lizana, I. Moreno, A. Márquez, C. Iemmi, E. Fernández, J. Campos and M. J. Yzuel, "Time fluctuations of the phase modulation in a liquid crystal on silicon display: characterization and effects in diffractive optics," Opt. Express 16, 16711-16722 (2008).

[13] A. Lizana, A. Márquez, L. Lobato, Y. Rodange, I. Moreno, C. Iemmi, and J. Campos, "The minimum Euclidean distance principle applied to improve the modulation diffraction efficiency in digitally controlled spatial light modulators," Opt. Express 18, 10581-10593 (2010).

[14] J. García-Márquez, J. E. A. Landgrave, N. Alcalá-Ochoa, and C. Pérez-Santos, "Recursive wavefront aberration correction method for LCoS spatial light modulators," Opt. Lasers Eng. 49(6), 743-748 (2011).

[15] L. Yao, Z. Zhiyao and W. Runwen, "Optical heterodyne measurement of the phase retardation of a quarter-wave plate," Opt. Let. 13, 553-555 (1988).

[16] L.-H. Shyu, C.-L.-Chen and D.-C. Su, "Method for measuring the retardation of a wave plate," Appl. Opt. 32, 42284230 (1993).

[17] A. Márquez, M. Yamauchi, J. A. Davis and D. J. Franich, "Phase measurements of a twisted nematic liquid crystal spatial light modulator with a common-path interferometer," Opt. Commun. 190, 129-133 (2001).

[18] S. Nakadate, "High precision retardation measurement using phase detection of Young's fringes," Appl. Opt. 29, $242-246$ (1990).

[19] H. G. Jerrard, "Transmission of light through birefringent and optically active media: the Poincaré sphere," J. Opt. Soc. Am. 44, 634-640 (1954).

[20] P. Kurzynowski, "Senarmont compensator for elliptically birefringent media," Opt. Commun. 197, 235-238 (2001).

[21] G. Goldstein, [Polarized Light], (Marcel Dekker, New York 2003).

[22] N. G. Theofanous, "Error analysis of circular polarizer-analyzer systems for phase retardation measurements," J. Opt. Soc. Am. A 4, 2191-2200 (1987).

[23] P.A. Williams, A.H. Rose and C.M. Wang, "Rotating-polarizer polarimeter for accurate retardance measurement," Appl. Opt. 36, 6466-6472 (1997).

[24] M. Born y E. Wolf, [Principles of Optics, Cambridge University], 7nth edition, Cambridge (1999) pp. 823-826.

[25] A. Márquez, C. Cazorla, M. J. Yzuel and J. Campos, "Characterization of the retardance of a wave plate to increase the robustness of amplitude-only and phase-only modulations of a liquid crystal display," J. Mod. Opt. 52, 633-650 (2005).

[26] A. Hermerschmidt, S. Osten, S. Krüger and Thomas Blümel, "Wave front generation using a phase-only modulating liquid-crystalbased micro-display with HDTV resolution," Proc. SPIE 6584, 65840E (2007).

[27] J. R. Moore, N. Collings, W. A. Crossland, A. B. Davey, M. Evans, A. M. Jeziorska, M. Komarčević, R. J. Parker, T. D. Wilkinson and H. Xu, "The silicon backplane design for an LCOS polarization-insensitive phase hologram SLM," IEEE Photon. Techol. Lett. 20, 60-62 (2008). 